\title{
Thermodynamics of gravitationally induced particle creation scenario in DGP braneworld
}

\author{
Abdul Jawad $^{\mathrm{a}}$, Shamaila Rani ${ }^{\mathrm{b}}$, Salman Rafique ${ }^{\mathrm{c}}$ \\ Department of Mathematics, COMSAT Institute of Information Technology, Lahore 54000, Pakistan
}

Received: 13 July 2017 / Accepted: 5 January 2018 / Published online: 29 January 2018

(C) The Author(s) 2018. This article is an open access publication

\begin{abstract}
In this paper, we discuss the thermodynamical analysis for gravitationally induced particle creation scenario in the framework of DGP braneworld model. For this purpose, we consider apparent horizon as the boundary of the universe. We take three types of entropy such as Bakenstein entropy, logarithmic corrected entropy and power law corrected entropy with ordinary creation rate $\Gamma$. We analyze the first law and generalized second law of thermodynamics analytically for these entropies which hold under some constraints. The behavior of total entropy in each case is also discussed which implies the validity of generalized second law of thermodynamics. Also, we check the thermodynamical equilibrium condition for two phases of creation rate, that is constant and variable $\Gamma$ and found its vitality in all cases of entropy.
\end{abstract}

\section{Introduction}

It is believed that the universe undergoes an accelerated expansion due to mysterious form of force called dark energy (DE) which was firstly predicted by two independent teams of cosmologists [1,2]. Both of them used distant type Ia supernova as standard candles to measure the expansion of the universe. This discovery was unexpected, because before this invention, cosmologists just think that the expansion of the universe would be decelerating because of the gravitational attraction of the matter in the universe. In the accelerated expansion of the universe, DE plays major role but its nature is still unknown. The simplest candidate of DE is the cosmological constant, but its composition and mechanism are unknown. More generally, the detail of its equation of state (EoS) and relationship with the standard model of particle

\footnotetext{
a e-mails: jawadab181@yahoo.com; abduljawad@ciitlahore.edu.pk

be-mail: shamailatoor.math@yahoo.com

ae-mail: salmanmath004@gmail.com
}

physics continue to be investigated both through observationally and theoretically [3]. In order to explain this cosmic acceleration, various DE models and modified theories of gravity have been developed such as $f(R)$ theory, $f(T)$ theory, Brans-Dicke theory, dynamical Chern-Simons modified gravity, DGP Braneworld model, etc.

The concept of thermodynamics in cosmological system originates through black hole physics. It was suggested [4] that the temperature of Hawking radiations emitting from black holes is proportional to their corresponding surface gravity on the event horizon. Jacobson [5] found a relation between thermodynamics and the Einstein field equations. He derived this relation on the basis of entropy-horizon area proportionality relation along with first law of thermodynamics (also called Clausius relation) $d Q=T d S$, where $d Q, T$ and $d S$ indicate the exchange in energy, temperature and entropy change for a given system. It was shown that the field equations for any spherically symmetric spacetime can be expressed as $T d S=d E+P d V(E, P$ and $V$ represent the internal energy, pressure and volume of the spherical system) for any horizon [6].

The generalized second law of thermodynamics (GSLT) has been studied extensively in the scenario of expanding behavior of the universe. The GSLT states that the entropy of matter inside the horizon plus entropy of the horizon remains positive and increases with the passage of time [7]. In order to discuss GSLT, horizon entropy of the universe can be taken as one quarter of its horizon area [8] or power law corrected [911] or logarithmic corrected [12] forms. Many people have explored the validity of GSLT of different systems including interaction of two fluid components like DE and dark matter [13-16], as well as interaction of three components of fluid [17-19] in the FRW universe by using simple horizon entropy of the universe. The thermodynamical analysis widely performed in modified theories of gravity [20-25].

The gravitationally induced particle creation is another well-known mechanism which was firstly introduced by 
Schrodinger [26] on microscopic level. This mechanism was further extended by Parker et al. towards quantum field theory in curved spacetimes [27-31]. The macroscopic description of particle creation mechanism induced by gravitational field was presented by Prigogine et al. [32]. Later on, the covariant description of this mechanism was developed [33,34] as well as the physical difference between particle creation and bulk viscosity was also given [35]. The particle creation process can be described with the inclusion of backreaction term in the Einstein field equations whose negative pressure may help in explaining the cosmic acceleration. In this way, various phenomenological models of particle creation have been presented [36-41]. It is also shown that phenomenological particle production [42-45] help in explaining the cosmic acceleration and paved the alternative way to the concordance $\Lambda$ CDM model.

The fact that we resides in a three-dimensional space embedded in an extra-dimensional world and fivedimensional models in which universe would be a hypersurface has attain a great attention. The four-dimensional Einsteins equations projected onto the brane have been explored by Shiromizu et al. [46]. The approaches which made on the basis of brane-world in the early-time cosmology favor a particular model of cosmic evolution featured by quadratic relations between the energy density and the Hubble parameter, dubbed quadratic cosmology $[47,48]$.

Recently, by assuming the gravitationally induced particle scenario with constant specific entropy and arbitrary particle creation rate $(\Gamma)$, thermodynamics on the apparent horizon for FRW universe has been discussed [49]. They have investigated the first law, GSLT and thermodynamical equilibrium by assuming the EoS for perfect fluid and put forward various constraints on $\Gamma$ for which thermodynamical laws hold. Our aim is to discuss the thermodynamical analysis on the apparent horizon for gravitationally induced particle creation scenario with ordinary creation rate $\Gamma$ by assuming entropies (Bakenstein entropy or usual entropy, logarithmic corrected entropy and power law corrected entropy) in DGP braneworld model. The scheme of the paper is as follows: In the next section, we will present the basic equations of DGP brane-world, particle creation rate and cosmological parameters. Sections 3, 4, 5 contain the discussion of thermodynamic quantities as well as its laws corresponding to usual, logarithmic and power law corrected entropies, respectively. The last section comprises of concluding remarks on our results.

\section{Basic equations}

A most particular version was proposed by Dvali et al. [50,51], in which the four dimensional FRW universe is enclosed in a five dimensional Minkoski bulk with infinite size. The gravitational laws were obtained by adding an
Einstein-Hilbert term to the action of brane computed with the brane's intrinsic curvature. The presence of such a term in the action is generically induced by quantum corrections coming from the bulk gravity and its coupling with matter living on the brane and must be included for a large class of theories for self-consistency [52,53]. Here, we consider 3-brane embedded in a $5 D$ space-time with an intrinsic curvature term included in the brane whose action can be written as

$S_{(5)}=-\frac{1}{2 \kappa^{2}} \int d^{5} X \sqrt{-\tilde{g}} \tilde{R}+\int d^{5} X \sqrt{-\tilde{g}} L_{m}$,

where $L_{m}$ is the brane curvature term, given by

$L_{m}=-\frac{1}{2 \mu^{2}} \int d^{4} x \sqrt{-g} R$,

and $\kappa^{2}=8 \pi G_{(5)}=M_{(5)}^{-3}, \mu^{2}=8 \pi G_{(4)}=M_{(4)}^{-2}$. The Eq. (1) represents the Einstein-Hilbert action in five dimensions for a five-dimensional metric $\tilde{g}_{\mathrm{AB}}$ (bulk metric) of Ricci scalar $\tilde{R}$. Similarly, Eq. (2) indicates the EinsteinHilbert action for the induced metric $\tilde{g}_{\mathrm{cd}}$ on the brane with $R$ appeared as its scalar curvature. From Eq. (1), we can get modified Friedmann equation as [54]

$H^{2}+\frac{k}{a^{2}}=\left(\sqrt{\frac{\rho}{3 M_{p}^{2}}+\frac{1}{4 r_{c}^{2}}}+\frac{\epsilon}{2 r_{c}}\right)^{2}$,

where $H=\frac{\dot{a}}{a}$ is Hubble parameter with $a(t)$ is the scale factor. Also, $\rho=\rho_{m}+\rho_{D}$, the subscripts $m$ and $D$ represent the energy densities corresponding to dark matter and DE respectively, $r_{c}$ is the crossover length which represents the scale that has length away from which gravity starts opening into the bulk [54]. Moreover it is the distance scale follow the comparison among $4 D$ and $5 D$ effects of gravity and can be written as [54]

$r_{\mathrm{c}}=\frac{M_{p}^{2}}{2 M_{5}^{3}}$,

where $M_{5}^{2}$ stands for the $5 D$ Planck mass and $M_{\mathrm{p}}^{2}$ is the $4 D$ Planck mass.

For the spatially flat DGP braneworld $(k=0)$, Eq. (3) reduces to

$H^{2}-\frac{\epsilon}{r_{\mathrm{c}}} H=\frac{\rho}{3 M_{p}^{2}}$.

There exist two different branches for the DGP model depending on the sign of $\epsilon$. These are as follows:

- For $\epsilon=+1$, there is a de Sitter solution for Eq. (5) with constant Hubble parameter, i.e., $H=\frac{1}{r_{c}} \Rightarrow a(t)=$ $a_{0} e^{\frac{t}{r_{c}}}$ in the absence of any kind of energy or matter field 
on the brane (i.e., $\rho=0$ ). However, this branch faces some problems like ghost instabilities [55].

- For $\epsilon=-1$, the accelerated expansion of the universe can only be explained through the inclusion of DE component in the DGP scenario.

We consider the latter case in the present work. The equation of continuity for this model will become

$\dot{\rho}+\Theta(\rho+P+\Pi)=0$,

where $\Pi$ is a particle creation pressure which represents the gravitationally induced process of particle creation and $\Theta=3 H$ is the fluid expansion. Differentiating Eq. (5) and replacing the value of $\dot{\rho}$ using Eq. (6), we obtain

$\dot{H}=\frac{-H(\rho+p+\Pi)}{M_{p}^{2}\left(2 H-\frac{\epsilon}{r_{c}}\right)}$.

The respective EoS for this model is giving by $p=(\gamma-1) \rho$ with $\frac{2}{3} \leq \gamma \leq 2$. The non-conservation of the total rate of change of number of particles, $N=n a^{3}$ with comoving volume $a^{3}$ and $n$ is the number density of particle production in an open thermodynamical system yields

$\dot{n}+\Theta n=n \Gamma$,

where $\Gamma$ is a particle creation rate has negative and positive phases. Negative $\Gamma$ represents the particle destruction and positive $\Gamma$ describes the elimination of particles. Furthermore, a non-zero $\Gamma$ produces effective bulk viscous pressure [33,35-37,56-58].

Now using the Eqs. (6), (8) and Gibbs relation, we get

$T d s=d\left(\frac{\rho}{n}\right)+p d\left(\frac{1}{n}\right)$.

An equation related to the creation pressure $\Pi$ and the creation rate $\Gamma$ has the form

$\Pi=-\frac{\Gamma}{\Theta}(\rho+p)$.

Under traditional assumption that the specific entropy of each particle is constant, i.e., the process is adiabatic or isentropic. This implies a dissipative fluid is similar to a perfect fluid with a non-conserved particle number. To discuss cosmological parameters, we insert Eq. (10) and $p=(\gamma-1) \rho$ in Eq. (7), to obtain

$\frac{\dot{H}}{H^{2}}=-\frac{3 \gamma\left(H-\frac{\epsilon}{r_{\mathrm{c}}}\right)\left(1-\frac{\Gamma}{3 H}\right)}{\left(2 H-\frac{\epsilon}{r_{c}}\right)}$.

The deceleration parameter $q$ can be written as

$q=-\frac{\dot{H}}{H^{2}}-1=\frac{3 \gamma\left(H-\frac{\epsilon}{r_{c}}\right)\left(1-\frac{\Gamma}{3 H}\right)}{\left(2 H-\frac{\epsilon}{r_{c}}\right)}-1$.
The effective EoS parameter for this model turns out to be

$\omega_{\mathrm{eff}}=\frac{p+\Pi}{\rho}=\gamma\left(1-\frac{\Gamma}{3 H}\right)-1$.

This parameter has ability to explain the different phases of the universe on the basis of $\Gamma$, i.e., if $\Gamma<3 H$, then we have quintessence era of the universe $\left(\omega_{\mathrm{eff}}<-1\right)$, if $\Gamma>3 H$ then effective EoS parameter represents the phantom era of the universe $\left(\omega_{\text {eff }}>-1\right)$ while for $\Gamma=3 H$, effective EoS parameter exhbits the cosmological constant $\left(\omega_{\text {eff }}=-1\right)$.

In the following, we will discuss first and second thermodynamical laws in the presence of particle creation rate $\Gamma$ on the apparent horizon.

\section{Thermodynamical analysis with usual entropy}

For flat FRW universe, Hubble parameter coincides with the apparent horizon as $R_{\mathrm{A}}=\frac{1}{H}$. Differentiating the apparent horizon with respect to time, we get

$\dot{R}_{A}=-\frac{\dot{H}}{H^{2}}=\frac{3 \gamma\left(H-\frac{\epsilon}{r_{c}}\right)\left(1-\frac{\Gamma}{3 H}\right)}{\left(2 H-\frac{\epsilon}{r_{c}}\right)}$.

The Bekenstein entropy and Hawking temperature of the apparent horizon are given by $(8 \pi=G=1)$

$S_{\mathrm{A}}=\frac{A}{4}=\frac{R_{A}^{2}}{8} \quad$ and $\quad T_{\mathrm{A}}=\frac{1}{2 \pi R_{A}}=\frac{4}{R_{A}}$,

where $A=4 \pi R_{A}^{2}$. The first law of thermodynamics at the horizon can be obtained through the Clausius relation as

$-d E_{\mathrm{A}}=T_{\mathrm{A}} d S_{\mathrm{A}}$.

For the sake of convenance, we consider $\Xi=T_{\mathrm{A}} d S_{\mathrm{A}}+d E_{\mathrm{A}}$. The differential $d E_{\mathrm{A}}$ is the amount of energy crossing the apparent horizon can be evaluated as [59]

$-d E_{\mathrm{A}}=\frac{1}{2} R_{\mathrm{A}}^{3}(\rho+p) H d t=\frac{3 \gamma\left(H-\frac{\epsilon}{r_{\mathrm{c}}}\right)}{2 H} d t$.

From Eq. (15), the differential of surface entropy at apparent horizon yields

$d S_{A}=\frac{3 \gamma}{4 H} \frac{\left(H-\frac{\epsilon}{r_{c}}\right)\left(1-\frac{\Gamma}{3 H}\right)}{\left(2 H-\frac{\epsilon}{r_{c}}\right)}$,

which leads to

$T_{\mathrm{A}} d S_{\mathrm{A}}=\frac{3 \gamma\left(H-\frac{\epsilon}{r_{\mathrm{c}}}\right)\left(1-\frac{\Gamma}{3 H}\right)}{\left(2 H-\frac{\epsilon}{r_{\mathrm{c}}}\right)} d t$.

Thus, $\Xi$ turns out to be

$\Xi=3 \gamma\left(H-\frac{\epsilon}{r}\right)\left(\frac{1-\frac{\Gamma}{3 H}}{2 H-\frac{\epsilon}{r_{c}}}-\frac{1}{2 H}\right)$. 
From this relation, it can be seen that first law of thermodynamics holds (i.e., $\Xi \rightarrow 0$ ) at the apparent horizon for $\Gamma=3 H\left(1-\frac{2 H-\frac{\epsilon}{r_{c}}}{2 H}\right)$.

Next, we will discuss the GSLT and thermodynamical equilibrium of a system containing perfect fluid distribution bounded by apparent horizon in DGG brane-world scenario. For GSLT, the total entropy of the system can not be decrease, i.e., $d\left(S_{\mathrm{A}}+S_{\mathrm{f}}\right) \geq 0$. In this relation, $S_{\mathrm{A}}$ and $S_{\mathrm{f}}$ appear as the entropy at apparent horizon and the entropy of cosmic fluid enclosed within the horizon, respectively. The Gibbs equation is given by

$T_{\mathrm{f}} d S_{\mathrm{f}}=d E_{\mathrm{f}}+p d V$,

where $T_{\mathrm{f}}$ is the temperature of the cosmic fluid and $E_{\mathrm{f}}$ is the energy of the fluid $\left(E_{\mathrm{f}}=\rho V\right)$. The evolution equation for fluid temperature having constant entropy can be described as [60]

$\frac{\dot{T}_{\mathrm{f}}}{T_{\mathrm{f}}}=(\Gamma-\Theta) \frac{\partial p}{\partial \rho}$.

Eq. (11) leads to $\Gamma-\Theta=\frac{\dot{H}\left(2 H-\frac{\epsilon}{r_{\mathrm{c}}}\right)}{\gamma H\left(H-\frac{\epsilon}{r_{\mathrm{c}}}\right)}$ which inserting in Eq. (22) gives the following equation

$$
\begin{aligned}
\ln \left(\frac{T_{\mathrm{f}}}{T_{0}}\right) & =\frac{2(\gamma-1)}{\gamma} \int \frac{d H}{H} \\
& \Rightarrow T_{\mathrm{f}}=T_{0}\left(H^{2}-\frac{\epsilon}{r_{\mathrm{c}}} H\right)^{\frac{(\gamma-1)}{\gamma}},
\end{aligned}
$$

where $T_{0}$ is the constant of integration. The differential of the fluid entropy can be obtained by using the Eq. (21) as follows

$$
\begin{aligned}
d S_{\mathrm{f}}= & -\frac{3 \gamma T_{0}^{-1}}{\left(2 H-\frac{\epsilon}{r_{\mathrm{c}}}\right)}\left(H-\frac{\epsilon}{r_{\mathrm{c}}}\right)\left(1-\frac{\Gamma}{3 H}\right)\left(H^{2}-\frac{\epsilon}{r_{\mathrm{c}}} H\right)^{\frac{1-\gamma}{\gamma}} \\
& \times\left(1-\frac{1}{2 H r_{\mathrm{c}}}-\frac{3 \gamma}{2 H}\left(H-\frac{\epsilon}{r_{\mathrm{c}}}\right)\right) d t
\end{aligned}
$$

Using Eqs. (18) and (24), we get the rate of change of total entropy as

$$
\begin{aligned}
\dot{S}_{T}= & \frac{3 \gamma\left(H-\frac{\epsilon}{r_{\mathrm{c}}}\right)\left(1-\frac{\Gamma}{3 H}\right)}{4 H\left(2 H-\frac{\epsilon}{r_{\mathrm{c}}}\right)}\left[1+4 T_{0}^{-1} H\left(H^{2}-\frac{\epsilon}{r_{\mathrm{c}}} H\right)^{\frac{1}{\gamma}-1}\right. \\
& \left.\times\left(\frac{1}{2 H} \frac{\epsilon}{r_{\mathrm{c}}}-\frac{3 \gamma}{2 H}\left(H-\frac{\epsilon}{r_{\mathrm{c}}}\right)-1\right)\right]
\end{aligned}
$$

where $S_{T}=S_{\mathrm{A}}+S_{\mathrm{f}}$. We discuss the validity of GSLT on the basis of $\Gamma$ such that

- $\Gamma<3 H$ : The GSLT holds if the following constraint

$$
1>4 T_{0}^{-1}\left(H^{2}-\frac{\epsilon}{r_{\mathrm{c}}} H\right)^{\frac{1}{\gamma}-1}\left(H-\frac{\epsilon}{2 r_{\mathrm{c}}}+\frac{3 \gamma}{2}\left(H-\frac{\epsilon}{r_{\mathrm{c}}}\right)\right)
$$

satisfies. This shows that the GSLT satisfies in the qunitessence era of the evolving universe.

- $\Gamma>3 H$ : For this case, we have the constraint

$$
1<4 T_{0}^{-1}\left(H^{2}-\frac{\epsilon}{r_{\mathrm{c}}} H\right)^{\frac{1}{\gamma}-1}\left(H-\frac{\epsilon}{2 r_{\mathrm{c}}}+\frac{3 \gamma}{2}\left(H-\frac{\epsilon}{r_{\mathrm{c}}}\right)\right),
$$

which implies the GSLT holds in phantom era of the universe.

- $\underline{\Gamma=3 H}$ : This case implies $\dot{S}_{T}=0$ in the cosmological constant era.

Replacing $d t$ to $\frac{d H}{\dot{H}}$ and integrating Eq. (25), we get

$$
\begin{aligned}
& S_{\mathrm{T}}=S_{\mathrm{A}}+S_{\mathrm{f}} \\
& =-\frac{1}{8 H^{2} \epsilon^{2} r_{c} T_{0}(1-\gamma)}\left(1-\frac{\epsilon}{H^{2} r_{c}}\right)^{\frac{-1}{\gamma}}\left(1-\frac{H^{2} r_{c}}{\epsilon}\right)^{\frac{-1}{\gamma}} \\
& {\left[6 \gamma^{2} \epsilon^{2}{ }_{2} F 1\left(\frac{-1+\gamma}{\gamma},-\frac{1}{\gamma}, 2-\frac{1}{\gamma}, \frac{\epsilon}{H^{2} r_{c}}\right)\right.} \\
& \left(H^{2}-\frac{\epsilon}{r_{c}}\right)^{\frac{1}{\gamma}}\left(1-\frac{H^{2} r_{c}}{\epsilon}\right)^{\frac{1}{\gamma}}+2\left(H^{2}-\frac{\epsilon}{r_{c}}\right)^{\frac{1}{\gamma}} \\
& \times r_{c}^{2}\left\{-4 H \epsilon(-1+\gamma){ }_{2} F 1\left(-\frac{1}{2},-\frac{1}{\gamma}, \frac{1}{2}, \frac{H^{2} r_{c}}{\epsilon},\right)\right. \\
& \times\left(1-\frac{\epsilon}{H^{2} r_{c}}\right)^{\frac{1}{\gamma}}+\gamma\left(6 H^{3}(-1+\gamma)\right. \\
& \times{ }_{2} F 1\left(\frac{1}{2}, \frac{-1+\gamma}{\gamma} \frac{3}{2}, \frac{H^{2} r_{c}}{\epsilon}\right)\left(1-\frac{\epsilon}{H^{2} r_{c}}\right)^{\frac{1}{\gamma}} \\
& \left.\left.+\epsilon_{2}^{2} F 1\left(\frac{-1+\gamma}{\gamma},-\frac{1}{\gamma}, 2-\frac{1}{\gamma}, \frac{\epsilon}{H^{2} r_{c}}\right)\left(1-\frac{H^{2} r_{c}}{\epsilon}\right)^{\frac{1}{\gamma}}\right)\right\} \\
& +2 H^{2} r_{c}^{3}\left(H^{2}-\frac{\epsilon}{r_{c}}\right)^{\frac{1}{\gamma}}(-1+\gamma)\left(4 H_{2} F 1\right. \\
& \times\left(\frac{1}{2}, \frac{-1+\gamma}{\gamma}, \frac{3}{2}, \frac{H^{2} r_{c}}{\epsilon}\right)\left(1-\frac{\epsilon}{H^{2} r_{c}}\right)^{\frac{1}{\gamma}} \\
& +\gamma \epsilon\left(-{ }_{2} F 1\left(-\frac{1}{\gamma},-\frac{1}{\gamma}, \frac{-1+\gamma}{\gamma}, \frac{\epsilon}{H^{2} r_{c}}\right)\right. \\
& \times\left(1-\frac{H^{2} r_{c}}{\epsilon}\right)^{\frac{1}{\gamma}}+\left(1-\frac{\epsilon}{H^{2} r_{c}}\right)^{\frac{1}{\gamma}}(-1 \\
& \left.\left.\left.+\left(1-\frac{H^{2} r_{c}}{\epsilon}\right)^{\frac{1}{\gamma}}\right)\right)\right)+(-1+\gamma) \epsilon \times r_{c}\left\{6 \gamma H\left(H^{2}-\frac{\epsilon}{r_{c}}\right)^{\frac{1}{\gamma}}\right. \\
& \times\left(-2\left(1-\frac{\epsilon}{H^{2} r_{c}}\right)^{\frac{1}{\gamma}}{ }_{2} F 1\left(-\frac{1}{2}, \frac{-1}{\gamma}, \frac{1}{2}, \frac{H^{2} r_{c}}{\epsilon}\right)\right. \\
& -H \gamma \times\left(1-\frac{H^{2} r_{c}}{\epsilon}\right)^{\frac{1}{\gamma}}{ }_{2} F 1\left(-\frac{1}{\gamma},-\frac{1}{\gamma}, \frac{-1+\gamma}{\gamma}, \frac{\epsilon}{H^{2} r_{c}}\right) \\
& +\left(1-\frac{\epsilon}{H^{2} r_{c}}\right)^{\frac{1}{\gamma}} \times\left(-1+\left(1-\frac{H^{2} r_{c}}{\epsilon}\right)^{\frac{1}{\gamma}}\right) \\
& \left.\left.-\epsilon\left(1-\frac{\epsilon}{H^{2} r_{c}}\right)^{\frac{1}{\gamma}}\left(1-\frac{H^{2} r_{c}}{\epsilon}\right)^{\frac{1}{\gamma}}\right\}\right] \text {. }
\end{aligned}
$$




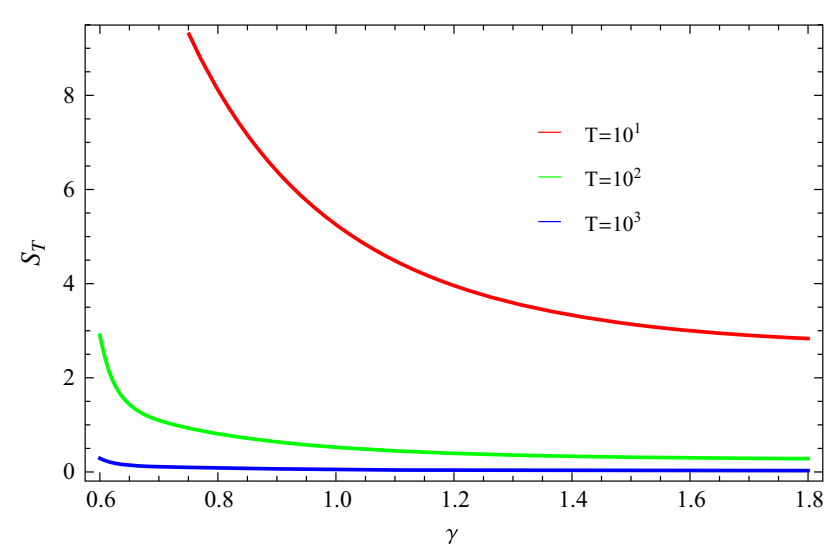

Fig. 1 Plot of total entropy versus $\gamma$

The plot between total entropy and parameter $\gamma$ is shown in Fig. 1 for three values of $T$ by setting constant values as $H=67, r_{c}=\frac{1}{67}, \epsilon=-1$. We observe that $S_{T} \geq 0$ for all the values of $T$ which leads to the validity of GSLT.

\subsection{Thermal equilibrium scenario}

Further, we will discuss the thermal equilibrium scenario in the present case. For thermodynamical equilibrium, the entropy function attains a maximum value and satisfies the condition $d^{2} S_{T}=d^{2}\left(S_{\mathrm{A}}+S_{\mathrm{f}}\right)<0$. For this purpose, we consider two cases of particle creation rate $(\Gamma)$.

\section{Case 1: $\underline{\Gamma=\text { constant }}$}

Firstly, we consider particle creation rate $\Gamma$ as a constant. Under this scenario, differentiating Eq. (25) w.r.t time, it results the following second order differential equation

$$
\begin{aligned}
\ddot{S_{T}}= & -\frac{3 \gamma\left(1-\frac{\Gamma}{3 H}\right)\left(-\frac{\epsilon}{r_{c}}+H\right) \lambda \dot{H}}{2 H\left(-\frac{\epsilon}{r_{c}}+2 H\right)^{2}}+\frac{3 \gamma\left(1-\frac{\Gamma}{3 H}\right) \lambda \dot{H}}{4 H\left(-\frac{\epsilon}{r_{c}}+2 H\right)} \\
& +\gamma \Gamma\left(1-\frac{\Gamma}{3 H}\right) \times \frac{\left(-\frac{\epsilon}{r_{c}}+H\right) \lambda \dot{H}}{4 H^{3}\left(-\frac{\epsilon}{r_{c}}+2 H\right)} \\
& -\frac{3 \gamma\left(1-\frac{\Gamma}{3 H}\right)\left(-\frac{\epsilon}{r_{c}}+H\right) \lambda \dot{H}}{4 H^{2}\left(-\frac{\epsilon}{r_{c}}+2 H\right)}+\frac{3 \gamma}{4 H\left(-\frac{\epsilon}{r_{c}}+2 H\right)} \\
& \times\left(1-\frac{\Gamma}{3 H}\right)\left(-\frac{\epsilon}{r_{c}}+H\right)\left(\frac{4\left(-\frac{\epsilon}{r_{c}}+H^{2}\right)^{\frac{1-\gamma}{\gamma}}}{T_{0}}\right.
\end{aligned}
$$

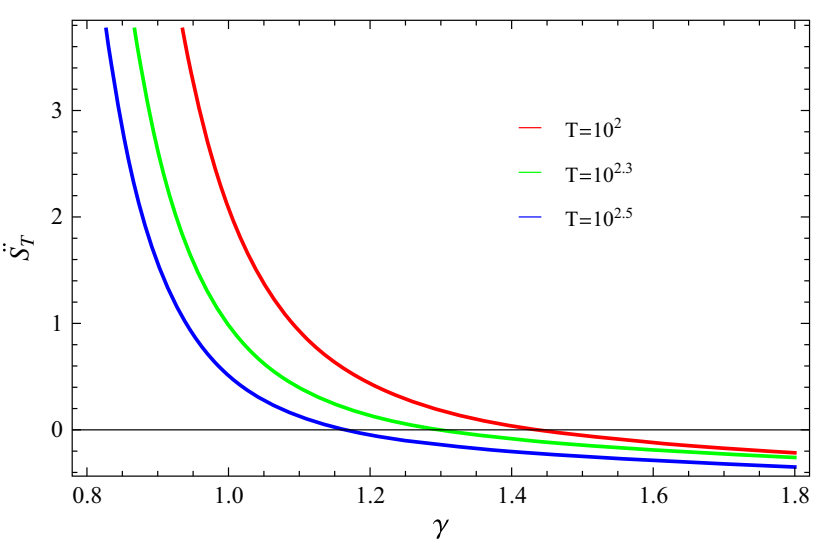

Fig. 2 Plot of $\ddot{S_{T}}$ versus $\gamma=$ constant

$$
\begin{aligned}
& \times\left(-1+\frac{\epsilon}{2 r_{c} H}-\frac{3 \gamma}{2 H} \times\left(-\frac{\epsilon}{r_{c}}+H\right)\right) \dot{H} \\
& +\frac{4(1-\gamma) H}{\gamma T_{0}}\left(-\frac{\epsilon H}{r_{c}}+H^{2}\right)^{-1+\frac{1-\gamma}{\gamma}} \\
& \times\left(-1+\frac{\epsilon}{2 H r_{c}}-\frac{3 \gamma\left(-\frac{\epsilon}{r_{c}}+H\right)}{2 H}\right)\left(-\frac{\epsilon}{r_{c}}+2 H \dot{H}\right) \\
& +4 H\left(-\frac{\epsilon H}{r_{c}}+H^{2}\right)^{\frac{1-\gamma}{\gamma}} \\
& \left.\times \frac{\left(-\frac{\epsilon \dot{H}}{2 r_{c} H^{2}}-\frac{3 \gamma \dot{H}}{2 H}+\frac{3 \gamma\left(-\frac{\epsilon}{r_{c}}+H\right)}{2 H^{2}}\right)}{T_{0}}\right) .
\end{aligned}
$$

where $\lambda=1+\frac{4 H}{T_{0}}\left(-\frac{\epsilon H}{r_{c}}+H^{2}\right)^{\frac{1-\gamma}{\gamma}}\left(-1+\frac{\epsilon}{2 r_{c} H}-\right.$ $\left.\frac{3 \gamma\left(-\frac{\epsilon}{r_{c}}+H\right)}{2 H}\right)$. The plot between $\ddot{S_{T}}$ versus $\gamma$ for three values of $T$ with constant values of $H=67, r_{c}=\frac{1}{67}$. $\epsilon=-1$, $q=-0.53$ as shown in Fig. 2. One can observe that the thermodynamical equilibrium condition holds for all values of $T$ with specific ranges of $\gamma$. For example, for $T=10^{2}$, thermodynamical equilibrium holds for the range $1.4<\gamma \leq 1.8$ and does not obey for $0.6 \leq \gamma \leq 1.4$. For $T=10^{2.3}$, thermal equilibrium holds for the range $1.3<\gamma \leq 1.8$ and does not showing the validity for $0.6 \leq \gamma \leq 1.3$. However, for $T=10^{2.5}$, thermodynamic equilibrium condition holds for the range $1.2<\gamma \leq 1.8$ and disobey for the range $0.6 \leq \gamma<1.2$.

Case 2: $\Gamma=\Gamma(t)$

Here we take $\Gamma$ as variable parameter, i.e., $\Gamma=\Gamma(t)$, for which Eq. (25) becomes 


$$
\begin{aligned}
& \ddot{S_{T}}=-\frac{3 \gamma \lambda\left(-\frac{\epsilon}{r_{c}}+H\right)\left(1-\frac{\Gamma}{3 H}\right) \dot{H}}{2 H\left(-\frac{\epsilon}{r_{c}}+2 H\right)^{2}} \\
& +\frac{3 \gamma \lambda\left(1-\frac{\Gamma}{3 H}\right) \dot{H}}{4 H\left(-\frac{\epsilon}{r_{c}}+2 H\right)}-3 \gamma \lambda\left(1-\frac{\Gamma}{3 H}\right) \dot{H} \\
& \times \frac{\left(-\frac{\epsilon}{r_{c}}+H\right)}{4 H^{2}\left(-\frac{\epsilon}{r_{c}}+2 H\right)}+\frac{3 \gamma\left(-\frac{\epsilon}{r_{c}}+H\right)\left(1-\frac{\Gamma}{3 H}\right)}{4 H\left(-\frac{\epsilon}{r_{c}}+2 H\right)} \\
& \times\left(4\left(-\frac{\epsilon H}{r_{c}}+H^{2}\right)^{\frac{1-\gamma}{\gamma}} \times \frac{\left(-1+\frac{\epsilon}{2 r_{c} H}-\frac{3 \gamma\left(-\frac{\epsilon}{r_{c}}+H\right)}{2 H}\right) \dot{H}}{T_{0}}\right. \\
& +\frac{4(1-\gamma) H}{\gamma T_{0}}\left(-\frac{\epsilon H}{r_{c}}+H^{2}\right)^{-1+\frac{1-\gamma}{\gamma}} 4 H(1-\gamma) \\
& \times\left(-1+\frac{\epsilon}{2 r_{c} H}-\frac{3 \gamma\left(-\frac{\epsilon}{r_{c}}+H\right)}{2 H}\right)\left(-\frac{\epsilon \dot{H}}{r_{c}}+2 H \dot{H}\right) \\
& +\frac{4 H\left(-\frac{\epsilon H}{r_{c}}+H^{2}\right)^{\frac{1-\gamma}{\gamma}}\left(-\frac{\epsilon \dot{H}}{2 r_{c} H^{2}}-\frac{3 \gamma \dot{H}}{2 H}+\frac{3 \gamma\left(-\frac{\epsilon}{r_{c}}+H\right) \dot{H}}{2 H^{2}}\right)}{T_{0}} \\
& +\frac{3 \gamma \lambda\left(-\frac{\epsilon}{r_{c}}+H\right)\left(\frac{\Gamma \dot{H}}{3 H^{2}}-\frac{\dot{\Gamma}}{3 H}\right)}{4 H\left(-\frac{\epsilon}{r_{c}}+2 H\right)},
\end{aligned}
$$

where

$\dot{\Gamma}=-\frac{9 \gamma\left(\frac{\Gamma}{3 H}\right)\left(H-\frac{\epsilon}{r_{c}}\right)\left(1-\frac{\Gamma}{3 H}\right)}{2 H-\frac{\epsilon}{r_{c}}}+3 H \Gamma\left(1-\frac{\Gamma}{3 H}\right)$.

The plot of $\ddot{S_{T}}$ versus $\gamma$ for three values of $T$ as shown in Fig. 3 by keeping the same constant values as in previous case. We observe that the thermodynamic equilibrium holds for all values of $T$ with different ranges of $\gamma$. For example, for $T=10^{2}$, thermodynamic equilibrium holds for the range $1.4<\gamma \leq 1.8$ and does not satisfying $0.6 \leq \gamma \leq 1.4$. For $T=10^{2.3}$, it leads to the validity of thermodynamic equilibrium for the range $1.3<\gamma \leq 1.8$ and does not valid for $0.6 \leq \gamma \leq 1.3$. However, for $T=10^{2.5}$, thermodynamic equilibrium holds for the range $1.2 \leq \gamma \leq 1.8$ and does not satisfying within $0.6 \leq \gamma<1.2$.

\section{Logarithmic corrected entropy}

Quantum gravity allows the logarithmic corrections in the presence of thermal equilibrium fluctuations and quantum fluctuations [61-67]. The logarithmic entropy corrections

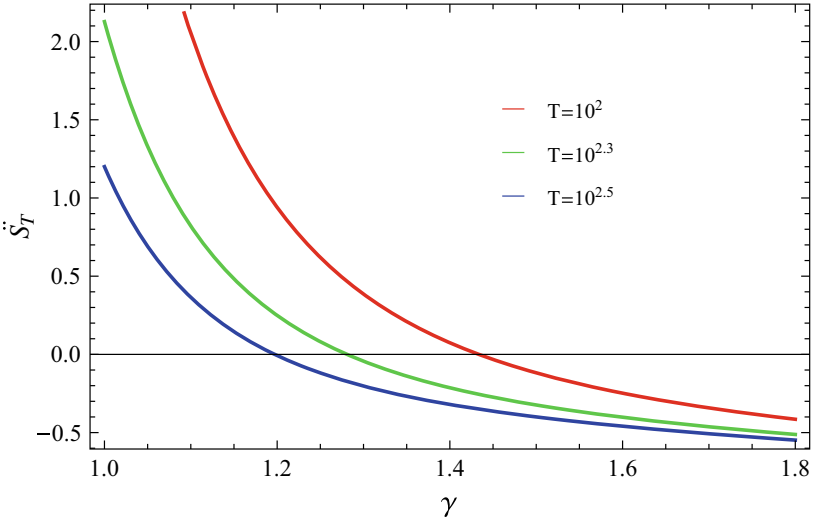

Fig. 3 Plot of $\ddot{S_{T}}$ versus $\gamma=\Gamma(t)$

can be defined as

$S_{\mathrm{A}}=\frac{A}{4 L_{\mathrm{p}}^{2}}+\alpha \ln \frac{A}{4 L_{\mathrm{p}}^{2}}+\beta \frac{4 L_{\mathrm{p}}^{2}}{A}$,

where $\alpha$ and $\beta$ are constants whose values are still under consideration. The differential form of above equation leads to

$$
\begin{aligned}
d S_{\mathrm{A}}= & -\frac{3 \gamma\left(H-\frac{\epsilon}{r_{\mathrm{c}}}\right)\left(1-\frac{\Gamma}{3 H}\right)}{\left(2 H-\frac{\epsilon}{r_{\mathrm{c}}}\right)} \\
& \times\left(\frac{1}{4 H L_{\mathrm{p}}^{2}}+2 \alpha H-16 \beta H^{3} L_{\mathrm{p}}^{2}\right) d t,
\end{aligned}
$$

which gives

$$
\begin{aligned}
T_{\mathrm{A}} d S_{\mathrm{A}}= & \frac{3 \gamma\left(H-\frac{\epsilon}{r_{\mathrm{c}}}\right)\left(1-\frac{\Gamma}{3 H}\right)}{\left(2 H-\frac{\epsilon}{r_{\mathrm{c}}}\right)} \\
& \times\left(\frac{1}{L_{\mathrm{p}}^{2}}+8 H^{2} \alpha-64 \beta H^{4} L_{\mathrm{p}}^{2}\right) d t .
\end{aligned}
$$

In view of this entropy, the quantity $\Xi$ takes the form

$$
\begin{aligned}
\Xi= & 3 \gamma\left(H-\frac{\epsilon}{r_{c}}\right)\left(-\frac{1}{2 H}+\frac{\left(1-\frac{\Gamma}{3 H}\right)}{\left(2 H-\frac{\epsilon}{r_{c}}\right)}\right. \\
& \left.\times\left(\frac{1}{L_{\mathrm{p}}^{2}}+8 H^{2} \alpha-64 \beta H^{4} L_{\mathrm{p}}^{2}\right)\right) .
\end{aligned}
$$

It can be observed from Eq. (32) that the first law of thermodynamics holds when $\Gamma=3 H\left(1-\frac{\left(2 H-\frac{\epsilon}{r_{c}}\right)}{2 H}\left(\frac{1}{L_{\mathrm{p}}^{2}}+8 H^{2} \alpha\right.\right.$ $\left.-64 \beta H^{4} L_{\mathrm{p}}^{2}\right)$ ). To discuss the GSLT for logarithmic corrected entropy of horizon, we obtain the total entropy by using Eqs. (24) and (30) as follows 


$$
\begin{aligned}
\dot{S_{T}}= & \frac{3 \gamma\left(H-\frac{\epsilon}{r_{\mathrm{c}}}\right)\left(1-\frac{\Gamma}{3 H}\right)}{\left(2 H-\frac{\epsilon}{r_{\mathrm{c}}}\right)}\left(4 H L_{\mathrm{p}}^{2}+2 \alpha H\right. \\
& -16 \beta H^{3} L_{\mathrm{p}}^{2}-T_{0}^{-1}\left(H^{2}-\frac{\epsilon}{r_{\mathrm{c}}} H\right)^{\frac{1-\gamma}{\gamma}} \\
& \left.\times\left(1-\frac{1}{2 H} \frac{\epsilon}{r_{\mathrm{c}}}-\frac{3 \gamma}{2 H}\left(H-\frac{\epsilon}{r_{\mathrm{c}}}\right)\right)\right) .
\end{aligned}
$$

The GSLT will hold under these constraints.

- For the case $\underline{\Gamma<3 H}$, the GSLT satisfy in the quintessence region of the universe if the following constraint holds

$$
\begin{aligned}
4 L_{\mathrm{p}}^{2}+2 \alpha & \\
> & 16 \beta H^{2} L_{\mathrm{p}}^{2}+\left(H T_{0}\right)^{-1}\left(1-\frac{1}{2 H} \frac{\epsilon}{r_{\mathrm{c}}}-\frac{3 \gamma}{2 H}\left(H-\frac{\epsilon}{r_{\mathrm{c}}}\right)\right) \\
& \times\left(H^{2}-\frac{\epsilon}{r_{\mathrm{c}}} H\right)^{\frac{1-\gamma}{\gamma}} .
\end{aligned}
$$

- $\underline{\Gamma>3 H}$ For this case, we obtain the following constraint

$$
\begin{aligned}
& 4 L_{\mathrm{p}}^{2}+2 \alpha \\
& <16 \beta H^{2} L_{\mathrm{p}}^{2}+\left(H T_{0}\right)^{-1}\left(1-\frac{1}{2 H} \frac{\epsilon}{r_{\mathrm{c}}}-\frac{3 \gamma}{2 H}\left(H-\frac{\epsilon}{r_{\mathrm{c}}}\right)\right) \\
& \quad \times\left(H^{2}-\frac{\epsilon}{r_{\mathrm{c}}} H\right)^{\frac{1-\gamma}{\gamma}} .
\end{aligned}
$$

which implies the GSLT holds in phantom era of the universe.

- The case $\underline{\Gamma=3 H}$ means $\dot{S}_{T}=0$ in the cosmological constant era.

The expression of total entropy in the form of Hubble parameter is given by

$$
\begin{aligned}
S_{\mathrm{T}} & =S_{\mathrm{A}}+S_{\mathrm{f}} \\
& =+8 H^{2} \beta L_{\mathrm{p}}^{2}-2 \ln (H)\left(\alpha+2 L_{\mathrm{p}}^{2}\right)+\frac{\gamma\left(H\left(H-\frac{\epsilon}{r_{c}}\right)\right)^{\frac{1}{\gamma}}}{2 H^{3} T_{0}} .
\end{aligned}
$$

The plot of total entropy $S_{T}$ versus $\gamma$ with respect to three values of $T$ is shown in Fig. 4 with constant values as $\alpha=-2$, $\beta=-0.00001, L_{p}=1$. It is observed that the total entropy is positive, i.e, $S_{T}>0$ which leads to the validity of GSLT for all values of $T$.

\subsection{Thermal equilibrium scenario}

Now we will discuss the thermodynamic equilibrium by assuming two cases for particle creation rate $\Gamma$ as follows:

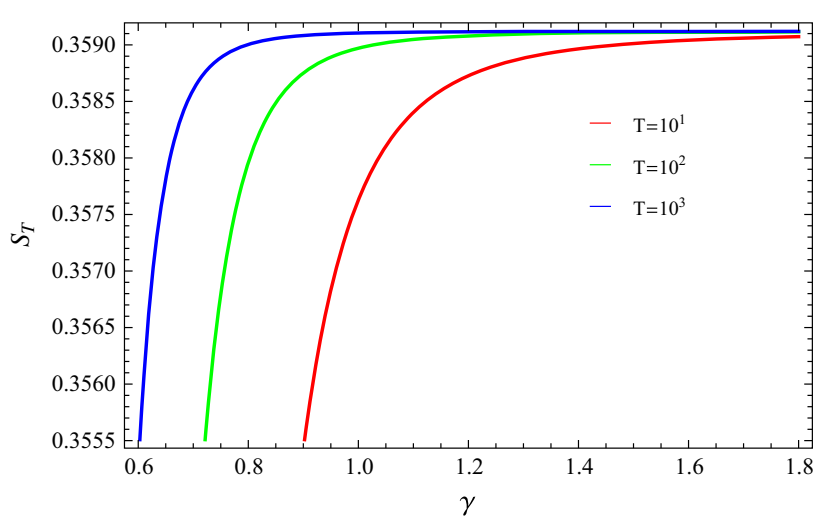

Fig. 4 Plots of total entropy versus $\gamma$

Case 1: $\underline{\Gamma=\text { constant }}$

In this way, the second order differential equation can be obtained from Eq. (33) for $\Gamma$ as a constant

$$
\begin{aligned}
& \ddot{S_{T}}=-\frac{6 \gamma\left(1-\frac{\Gamma}{3 H}\right)\left(H-\frac{\epsilon}{r_{c}}\right)}{\left(2 H-\frac{\epsilon}{r_{c}}\right)}\left(4 H L_{\mathrm{p}}^{2}-\lambda_{2}\right. \\
& \left.+2 \alpha H-16 L_{\mathrm{p}}^{2} \beta H^{3}\right) \dot{H}+3 \gamma \\
& \times \frac{\left(1-\frac{\Gamma}{3 H}\right)\left(4 H L_{\mathrm{p}}^{2}-\lambda_{2}+2 \alpha H-16 L_{\mathrm{p}}^{2} \beta H^{3}\right) \dot{H}}{\left(2 H-\frac{\epsilon}{r_{c}}\right)} \\
& +\gamma \Gamma\left(H-\frac{\epsilon}{r_{c}}\right) \frac{\left(4 H L_{\mathrm{p}}^{2}-\lambda_{2}+2 \alpha H-16 L_{\mathrm{p}}^{2} \beta H^{3}\right)}{H^{2}\left(2 H-\frac{\epsilon}{r_{c}}\right)} \\
& +\frac{3 \gamma\left(1-\frac{\Gamma}{3 H}\right)\left(H-\frac{\epsilon}{r_{c}}\right)}{\left(2 H-\frac{\epsilon}{r_{c}}\right)}\left(4 L_{\mathrm{p}}^{2} \dot{H}\right. \\
& -\frac{(1-\gamma)\left(1-\frac{3 \gamma\left(H-\frac{\epsilon}{r_{c}}\right)}{2 H}-\frac{\epsilon}{2 H r_{c}}\right)\left(H^{2}-\frac{\epsilon H}{r_{c}}\right)^{-1+\frac{1-\gamma}{\gamma}}}{\gamma T_{0}} \\
& \times\left(2 H \dot{H}-\frac{\epsilon \dot{H}}{r_{c}}\right) \\
& -\frac{\left(H^{2}-\frac{\epsilon}{r_{c}}\right)^{\frac{1-\gamma}{\gamma}}\left(-\frac{3 \gamma \dot{H}}{2 H}+\frac{3 \gamma\left(H-\frac{\epsilon}{r_{c}}\right)}{2 H^{2}}+\frac{\epsilon \dot{H}}{2 H^{2} r_{c}}\right)}{T_{0}} \\
& \left.+2 \alpha \dot{H}-48 L_{\mathrm{p}}^{2} \beta H^{2} \beta \dot{H}\right)
\end{aligned}
$$

where

$\lambda_{2}=\frac{\left(1-\frac{3 \gamma\left(H-\frac{\epsilon}{r_{c}}\right)}{2 H}-\frac{\epsilon}{2 H r_{c}}\right)\left(H^{2}-\frac{\epsilon H}{r_{c}}\right)^{\frac{1-\gamma}{\gamma}}}{T_{0}}$. 


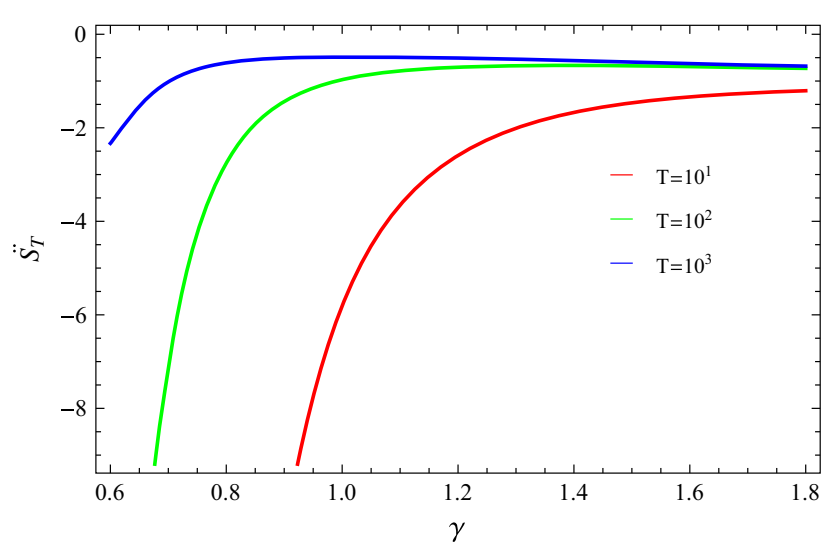

Fig. 5 Plot of $\ddot{S_{T}}$ versus $\gamma$ when $\Gamma=$ constant

The plot between $\ddot{S}_{T}$ and $\gamma$ for three values of $T$ by fixing the constant values $q=-0.53, \alpha=-2, \beta=-0.000000001$ and $L_{p}=1$ as shown in Fig. 5. It can be seen that thermodynamical equilibrium is obeying the condition $\ddot{S}_{T}<0$ for all values of $T$ which leads to the thermal equilibrium.

Case 2: $\Gamma=\Gamma(t)$

Taking $\Gamma$ as a function of $t$, Eq. (33) yields

$$
\begin{aligned}
\ddot{S_{T}}= & -\frac{6 \gamma\left(1-\frac{\Gamma}{3 H}\right)\left(H-\frac{\epsilon}{r_{c}}\right)}{\left(2 H-\frac{\epsilon}{r_{c}}\right)}\left(4 H L_{\mathrm{p}}^{2}-\lambda_{2}+2 \alpha H\right. \\
& \left.-16 L_{\mathrm{p}}^{2} \beta H^{3}\right) \dot{H}+3 \gamma \\
& \times \frac{\left(1-\frac{\Gamma}{3 H}\right)\left(4 H L_{\mathrm{p}}^{2}-\lambda_{2}+2 \alpha H-16 L_{\mathrm{p}}^{2} \beta H^{3}\right) \dot{H}}{\left(2 H-\frac{\epsilon}{r_{c}}\right)} \\
& +\frac{3 \gamma\left(1-\frac{\Gamma}{3 H}\right)}{\left(2 H-\frac{\epsilon}{r_{c}}\right)}\left(H-\frac{\epsilon}{r_{c}}\right) \\
& \times\left(4 L_{\mathrm{p}}^{2} \dot{H}-\frac{\left(1-\frac{3 \gamma\left(H-\frac{\epsilon}{r_{c}}\right)}{2 H}-\frac{\epsilon}{2 H r_{c}}\right)\left(H^{2}-\frac{\epsilon H}{r_{c}}\right)^{-1+\frac{1-\gamma}{\gamma}}}{\gamma T_{0}}\right. \\
& \times(1-\gamma)\left(2 H \dot{H}-\frac{\epsilon \dot{H}}{r_{c}}\right) \\
& -\frac{\left(-\frac{3 \gamma \dot{H}}{2 H}+\frac{3 \gamma\left(H-\frac{\epsilon}{r_{c}}\right)}{2 H^{2}}+\frac{\epsilon \dot{H}}{2 H^{2} r_{c}}\right)}{T_{0}} \\
& \left.\times\left(H^{2}-\frac{\epsilon}{r_{c}}\right)^{\frac{1-\gamma}{\gamma}}+2 \alpha \dot{H}-48 L_{\mathrm{p}}^{2} \beta H^{2} \beta \dot{H}\right) \\
& +3 \gamma\left(H-\frac{\epsilon}{r_{c}}\right) \\
& \times \frac{1}{\left(2 H-\frac{\epsilon}{r_{c}}\right)}\left(4 H L_{\mathrm{p}}^{2}-\lambda_{2}+2 \alpha H-16 L_{\mathrm{p}}^{2} \beta H^{3}\right) \\
& \times\left(\frac{\Gamma \dot{H}}{3 H^{2}}-\frac{\dot{\Gamma}}{3 H}\right) .
\end{aligned}
$$

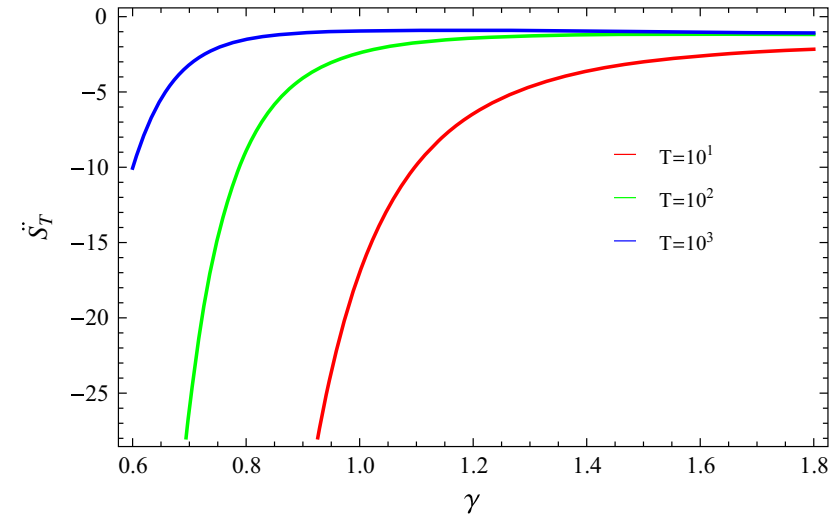

Fig. 6 Plot of $\ddot{S_{T}}$ versus $\gamma$ when $\Gamma=\Gamma(t)$

Figure 6 reperesents the plot between $\ddot{S_{T}}$ and $\gamma$ for three values of $T$ for variable $\Gamma$ for same constant values. Figure 6 indicates that the trajectories of $\ddot{S}_{T}$ corresponding to all the values of $T$ ensure the validity of thermodynamical equilibrium.

\section{Power law corrected entropy}

The power-law correction to the entropy-area law comes from association of the wave-function of the scalar field between the ground state and the exited state [68-72]. The correction term is also more significant for higher excitations. It is important to note that the correction term decreases faster with A and hence in the semi-classical limit (large area) the entropy-area law is recovered. The power entropy can $b$ given as

$S_{\mathrm{A}}=\frac{A}{4 L_{\mathrm{p}}^{2}}\left(1-K_{\delta} A^{1-\frac{\delta}{2}}\right), \quad K_{\delta}=\frac{\delta(4 \pi)^{\frac{\delta}{2}-1}}{(4-\delta) r_{c}^{4-\delta}}$

where $\delta$ is dimensionless constant and $r_{c}$ is the crossover scale. From Eq. (37) the differential of surface entropy at horizon can be expressed as

$$
\begin{aligned}
d S_{\mathrm{A}}= & \frac{3 \gamma\left(H-\frac{\epsilon}{r_{c}}\right)\left(1-\frac{\Gamma}{3 H}\right)}{\left(2 H-\frac{\epsilon}{r_{c}}\right)} \\
& \times\left(\frac{1}{4 H L_{\mathrm{p}}^{2}}-\frac{K_{\delta}}{4 H L_{\mathrm{p}}^{2}}\left(2-\frac{\delta}{2}\right)\left(\frac{1}{H}\right)^{3-\delta}\right) d t,
\end{aligned}
$$

which gives

$$
\begin{aligned}
T_{\mathrm{A}} d S_{\mathrm{A}}= & \frac{3 \gamma\left(H-\frac{\epsilon}{r_{c}}\right)\left(1-\frac{\Gamma}{3 H}\right)}{\left(2 H-\frac{\epsilon}{r_{c}}\right)} \\
& \times\left(\frac{1}{L_{\mathrm{p}}^{2}}-\left(2-\frac{\delta}{2}\right) K_{\delta} R^{2-\delta}\right) d t .
\end{aligned}
$$


In this entropy correction, we have

$$
\begin{aligned}
\Xi= & 3 \gamma\left(H-\frac{\epsilon}{r_{c}}\right)\left(-\frac{1}{2 H}+\frac{\left(1-\frac{\Gamma}{3 H}\right)}{\left(2 H-\frac{\epsilon}{r_{c}}\right)}\right. \\
& \left.\times\left(\frac{1}{L_{\mathrm{p}}^{2}}-\left(2-\frac{\delta}{2}\right) K_{\delta} R^{2-\delta}\right)\right),
\end{aligned}
$$

This equation shows that the first law of thermodynamics (i.e., $\Xi=0)$ holds for $\Gamma=3 H\left(1-\frac{\left(2 H-\frac{\epsilon}{r_{C}}\right)}{2 H\left(\frac{1}{L_{\mathrm{p}}^{2}}-\left(2-\frac{\delta}{2}\right) K_{\delta} R^{2-\delta}\right)}\right)$.

In the presence of power law corrected entropy, the rate of change of total entropy takes the form

$$
\begin{aligned}
\dot{S_{T}}= & \frac{3 \gamma\left(H-\frac{\epsilon}{r_{c}}\right)\left(1-\frac{\Gamma}{3 H}\right)}{\left(2 H-\frac{\epsilon}{r_{c}}\right)}\left(\frac{1}{4 H L_{\mathrm{p}}^{2}}-\frac{K_{\delta}}{4 H L_{\mathrm{p}}^{2}}\left(2-\frac{\delta}{2}\right)\left(\frac{1}{H}\right)^{3-\delta}\right. \\
& \left.-T_{0}^{-1}\left(H^{2}-\frac{\epsilon}{r_{c}} H\right)^{\frac{1-\gamma}{r_{c}}}\left(1-\frac{1}{2 H} \frac{\epsilon}{r_{c}}-\frac{3 \gamma}{2 H}\left(H-\frac{\epsilon}{r}\right)\right)\right) .
\end{aligned}
$$

To discuss the validity of GSLT, the following constraints must satisfy. These are as

- The condition $\underline{\Gamma<3 H}$ implies that

$$
\begin{aligned}
\frac{1}{4 L_{\mathrm{p}}^{2}}> & \frac{K_{\delta}}{4 L_{\mathrm{p}}^{2}}\left(2-\frac{\delta}{2}\right)\left(\frac{1}{H}\right)^{3-\delta}+H T_{0}^{-1} \\
& \times\left[1-\frac{1}{2 H} \frac{\epsilon}{r_{c}}-\frac{3 \gamma}{2 H}\left(H-\frac{\epsilon}{r}\right)\right] \times\left(H^{2}-\frac{\epsilon}{r_{c}} H\right)^{\frac{1-\gamma}{r_{c}}}
\end{aligned}
$$

- $\underline{\Gamma>3 H}$ For this case, we obtain the following constraint which indicates the validity of GSLT in phantom phase. It is given as

$$
\begin{aligned}
\frac{1}{4 L_{\mathrm{p}}^{2}}< & \frac{K_{\delta}}{4 L_{\mathrm{p}}^{2}}\left(2-\frac{\delta}{2}\right)\left(\frac{1}{H}\right)^{3-\delta}+H T_{0}^{-1} \\
& \times\left[1-\frac{1}{2 H} \frac{\epsilon}{r_{c}}-\frac{3 \gamma}{2 H}\left(H-\frac{\epsilon}{r}\right)\right] \times\left(H^{2}-\frac{\epsilon}{r_{c}} H\right)^{\frac{1-\gamma}{r_{c}}}
\end{aligned}
$$

- The case $\underline{\Gamma=3 H}$ gives $\dot{S}_{T}=0$ in the cosmological constant era.

The Eq. (41) in terms of Hubble parameter takes the following form

$$
\begin{aligned}
S_{T} & =S_{\mathrm{A}}+S_{\mathrm{f}} \\
& =\frac{1}{4}\left(\frac{1}{2 H^{2} L_{\mathrm{p}}^{2}}-\frac{\left(\frac{1}{H}\right)^{4-\delta} K_{\delta}}{2 L_{\mathrm{p}}^{2}}+\frac{2 \gamma\left(H\left(H-\frac{\epsilon}{r_{c}}\right)\right)^{\frac{1}{\gamma}}}{H^{3} T_{0}}\right) .
\end{aligned}
$$

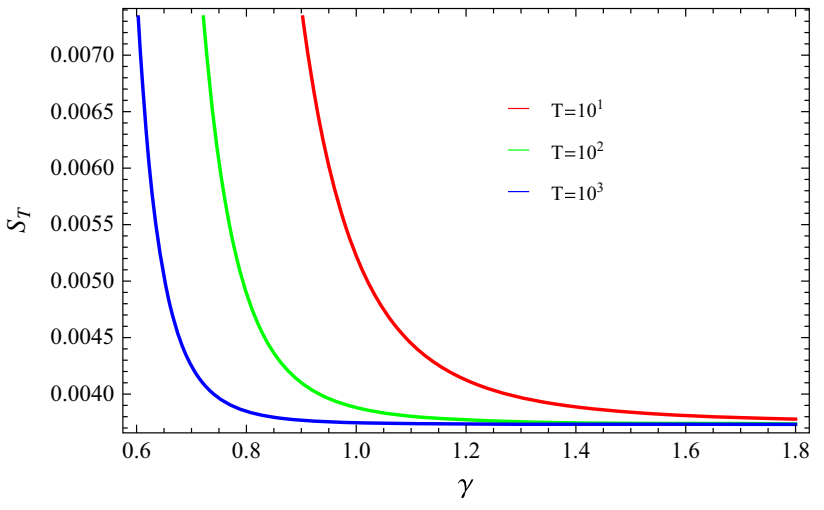

Fig. 7 Plot of total entropy versus $\gamma$

The plot of total entropy $\left(S_{T}\right)$ is shown in Fig. 7 versus $\gamma$ for three values of $T$ by assigning the constant values as $L=1$, $H=67, \frac{1}{67}, \alpha=-2$, and $\epsilon=-1$. We observe that total entropy is positive, i.e, $\left(S_{T}\right)>0$ which leads to the validity of GSLT for all values of $T$.

\subsection{Thermal equilibrium scenario}

For thermodynamic equilibrium, we assume two cases of $\Gamma$, i.e., $\Gamma$ is constant and $\Gamma$ is variable.

Case 1: $\underline{\Gamma=\text { constant }}$

For constant $\Gamma$, the second order differential equation of total entropy leads to

$$
\begin{aligned}
\ddot{S_{T}}= & -\frac{6 \gamma\left(1-\frac{\Gamma}{3 H}\right)\left(H-\frac{\epsilon}{r_{c}}\right) \lambda_{3}}{\left(2 H-\frac{\epsilon}{r_{c}}\right)^{2}+\frac{3 \gamma\left(1-\frac{\Gamma}{3 H}\right) \lambda_{3}}{\left(2 H-\frac{\epsilon}{r_{c}}\right)}} \\
& +\frac{\gamma \Gamma\left(H-\frac{\epsilon}{r_{c}}\right) \lambda_{3}}{H^{2}\left(2 H-\frac{\epsilon}{r_{c}}\right)}+\frac{3 \gamma\left(1-\frac{\Gamma}{3 H}\right)\left(H-\frac{\epsilon}{r_{c}}\right)}{\left(2 H-\frac{\epsilon}{r_{c}}\right)} \\
& \times\left(-\frac{\dot{H}}{4 H^{2} L_{\mathrm{p}}^{2}}+\frac{(3-\delta)\left(2-\frac{\delta}{2}\right)\left(\frac{1}{H}\right)^{4-\delta} K_{\delta} \dot{H}}{4 L_{\mathrm{p}}^{2}}\right. \\
& -\frac{(1-\gamma)\left(1-\frac{3 \gamma\left(H-\frac{\epsilon}{r_{c}}\right)}{2 H}-\frac{\epsilon}{2 H r_{c}}\right)\left(H^{2}-\frac{\epsilon H}{r_{c}}\right)^{-1+\frac{1-\gamma}{\gamma}}\left(2 H \dot{H}-\frac{\epsilon \dot{H}}{r_{c}}\right)}{\gamma T_{0}} \\
& \left.-\frac{\left(H^{2}-\frac{\epsilon H}{r_{c}}\right)^{\frac{1-\gamma}{\gamma}}\left(-\frac{3 \gamma \dot{H}}{2 H}+\frac{3 \gamma\left(H-\frac{\epsilon}{r_{c}}\right) \dot{H}}{2 H^{2}}+\frac{\epsilon \dot{H}}{2 r_{c} h^{2}}\right)}{T_{0}}\right),
\end{aligned}
$$

where

$$
\begin{aligned}
\lambda_{3}= & \left(\frac{1}{4 H L_{\mathrm{p}}^{2}}-\frac{\left(2-\frac{\delta}{2}\right)\left(\frac{1}{H}\right)^{3-\delta} K_{\delta}}{4 L_{\mathrm{p}}^{2}}-\left(1-\frac{3 \gamma\left(H-\frac{\epsilon}{r_{c}}\right)}{2 H}\right.\right. \\
& \left.\left.-\frac{\epsilon}{2 H r_{c}}\right) \times \frac{\left(H^{2}-\frac{\epsilon H}{r_{c}}\right)^{\frac{1-\gamma}{\gamma}}}{T_{0}}\right) \dot{H} .
\end{aligned}
$$




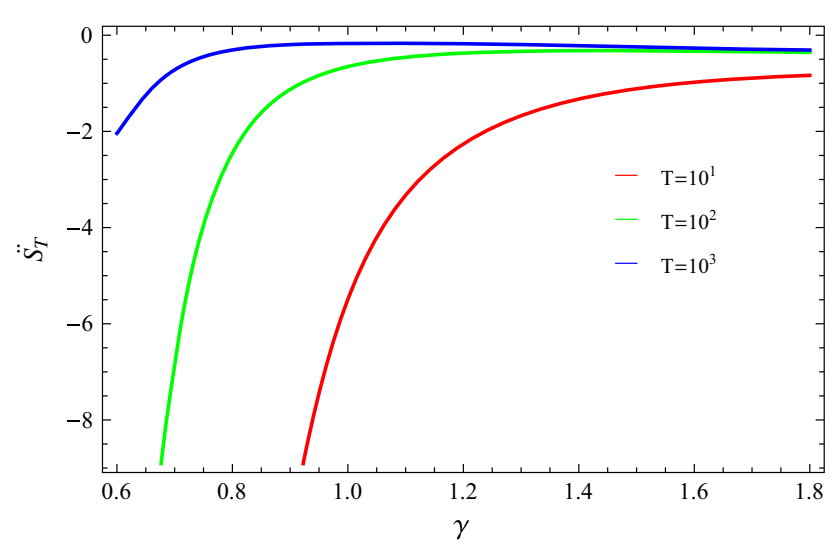

Fig. 8 Plot of $\ddot{S_{T}}$ versus $\gamma$

The graphical behavior of $\ddot{S_{T}}$ against $\gamma$ is shown in Figure 8 for three values of $T$ by keeping the constant values as $H=$ $67, r_{c}=\frac{1}{67}, \epsilon=-1, q=-0.53, \delta=-2, L=1$. It is found that the trajectories of this plot satisfy the condition $\ddot{S_{T}}<$ 0 for all values of $T$ which leads to the thermodynamical equilibrium of the system.

Case 2: $\Gamma=\Gamma(t)$

For variable $\Gamma$, second order differential equation of total entropy turns out to be

$$
\begin{aligned}
\ddot{S_{T}}= & -\frac{6 \gamma\left(1-\frac{\Gamma}{3 H}\right)\left(H-\frac{\epsilon}{r_{c}}\right) \lambda_{3}}{\left(2 H-\frac{\epsilon}{r_{c}}\right)^{2}}+\frac{3 \gamma\left(1-\frac{\Gamma}{3 H}\right) \lambda_{3}}{\left(2 H-\frac{\epsilon}{r_{c}}\right)} \\
& +\frac{3 \gamma\left(1-\frac{\Gamma}{3 H}\right)\left(H-\frac{\epsilon}{r_{c}}\right)}{\left(2 H-\frac{\epsilon}{r_{c}}\right)} \\
& \times\left(-\frac{\dot{H}}{4 H^{2} L_{\mathrm{p}}^{2}}+\frac{(3-\delta)\left(2-\frac{\delta}{2}\right)\left(\frac{1}{4 H}\right)^{4-\delta} K_{\delta} \dot{H}}{4 L_{\mathrm{p}}^{2}}\right. \\
& -\left(2 H \dot{H}-\frac{\epsilon \dot{H}}{r_{c}}\right) \\
& \times \frac{(1-\gamma)\left(1-\frac{3 \gamma\left(H-\frac{\epsilon}{r_{c}}\right)}{2 H}-\frac{\epsilon}{2 H r_{c}}\right)\left(H^{2}-\frac{\epsilon H}{r_{c}}\right)^{-1+\frac{1-\gamma}{\gamma}}}{\gamma T_{0}} \\
& \left.-\left(H^{2}-\frac{\epsilon H}{r_{c}}\right)^{\frac{1-\gamma}{\gamma}} \frac{\left(-\frac{3 \gamma \dot{H}}{2 H}+\frac{3 \gamma\left(H-\frac{\epsilon}{r_{c}}\right) \dot{H}}{2 H^{2}}+\frac{\epsilon \dot{H}}{2 H^{2} r_{c}}\right)}{T_{0}}\right) \\
& +\frac{3 \gamma\left(H-\epsilon / r_{c}\right) \lambda_{3}\left(\frac{\Gamma \dot{H}}{3 H^{2}}-\frac{\dot{\Gamma}}{3 H}\right)}{2 H-\frac{\epsilon}{r_{c}}} .
\end{aligned}
$$

The plot between $\ddot{S_{T}}$ and $\gamma$ for three values of $T$ by setting the constant values as $H=67, r_{c}=\frac{1}{67}, \epsilon=-1, q=$

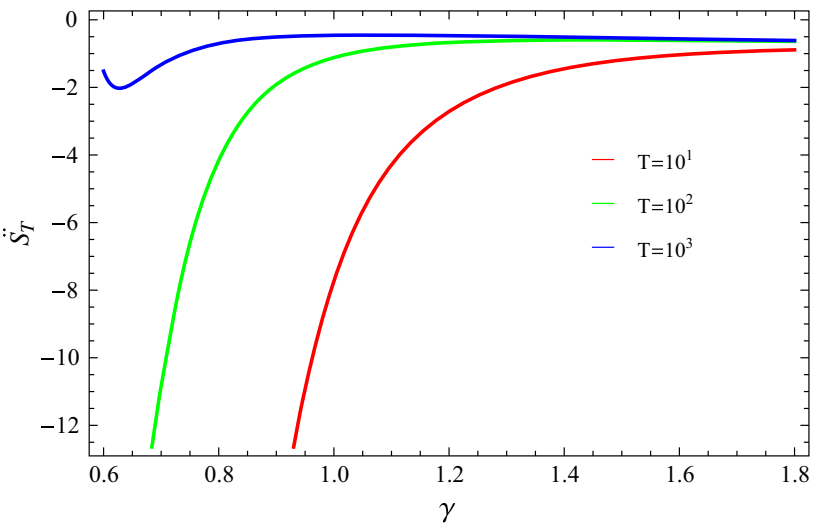

Fig. 9 Plot of $\ddot{S_{T}}$ versus $\gamma$

$-0.53, \delta=-2, L=1$ as shown in Fig. 9. We observe that thermodynamical equilibrium satisfying the condition $\ddot{S_{T}}<0$ for all values of $T$ which leads to thermodynamical disequilibrium of the system.

\section{Comparison and conclusion}

We have studied the thermodynamics on the apparent horizon for gravitationally induced particle creation scenario by assuming entropy corrections (Bakenstein entropy, logarithmic corrected entropy and power law corrected entropy) in DGP braneworld gravity. Considering the perfect fluid EoS $p=(\gamma-1) \rho$, we have analyzed the first law of thermodynamics, GSLT and thermodynamic equilibrium. We have fixed model parameters in the way to obtain the validity of GSLT. However, the results may be changed for other model parameters. The results have been summarized as follows:

For Bakenstein entropy, the first law of thermodynamics holds at apparent horizon when $\Gamma=3 H\left(1-\frac{\left(2 H-\frac{\epsilon}{r_{c}}\right)}{2 H}\right)$. The GSLT valid under some constraints in different eras of the evolving universe. In terms of total entropy scenario, GSLT remains valid for all values of $T$ with $\frac{2}{3} \leq \gamma \leq 2$ (Fig. 1). In the presence of usual entropy of horizon and for constant $\Gamma$, thermodynamical equilibrium holds for all values of $T$ with specific ranges of $\gamma$. For example, thermodynamical equilibrium is satisfying the condition $\frac{d^{2} S_{T}}{d t^{2}}<0$ for $1.4<$ $\gamma \leq 1.8,3<\gamma \leq 1.8,1.2<\gamma \leq 1.8$ and does not showing the validity $0.6 \leq \gamma \leq 1.4,0.6 \leq \gamma \leq 1.3$ and $0.6 \leq \gamma<1.2$ with $T=10^{2}, T=10^{2.3}$ and $T=10^{2.5}$ respectively (Fig. 2). For variable $\Gamma$, Fig. 3 indicates that thermodynamical equilibrium holds for all values of $T$ with different ranges of $\gamma$ such as $1.4<\gamma \leq 1.8,1.3<\gamma \leq 1.8$, $1.2 \leq \gamma \leq 1.8$ and does not obey $0.6 \leq \gamma \leq 1.4,0.6 \leq$ $\gamma \leq 1.3$ and $0.6 \leq \gamma<1.2$ for $T=10^{2}, T=10^{2.3}$ and $T=10^{2.5}$ respectively. 
For logarithmic corrected entropy, it has observed that first law of thermodynamics holds at apparent horizon when $\Gamma=3 H\left(1-\frac{\left(2 H-\frac{\epsilon}{r_{c}}\right)}{2 H}\left(\frac{1}{L_{\mathrm{p}}^{2}}+8 H^{2} \alpha-64 \beta H^{4} L_{\mathrm{p}}^{2}\right)\right)$. The GSLT valid under some constraints in different eras of the evolving universe. In terms of total entropy scenario, GSLT is valid for all values of $T$ with $\frac{2}{3} \leq \gamma \leq 2$ (Fig. 4). Thermodynamical equilibrium condition $\left(\frac{d^{2} S_{T}}{d t^{2}}<0\right)$ satisfied for all values of $T$ with all values of $\gamma$ for constant as well as variable $\Gamma$ (Figs. 5 and 6).

For power law entropy corrected, it has been observed that the first law of thermodynamics satisfies under constraint $\Gamma=3 H\left(1-\frac{\left(2 H-\frac{\epsilon}{r_{c}}\right)}{2 H\left(\frac{1}{L_{\mathrm{p}}^{2}}-\left(2-\frac{\delta}{2}\right) K_{\delta} R^{2-\delta}\right)}\right)$ and GSLT holds under some conditions on model parameters in quintessence, phantom and cosmological constant phases. Also, GSLT meets for all values of $T$ in the range $\left(\frac{2}{3} \leq \gamma \leq 2\right)$ (Fig. 7). It is found that, for constant as well as variable $\Gamma$, the thermodynamic equilibrium condition $\frac{d^{2} S_{T}}{d t^{2}}<0$ obey for all values of $T$ which leads to the thermodynamical equilibrium of the system.

Here we provide some details about past works and compare with underlying work. Harko et al. [73] considered the possibility of a gravitationally induced particle production through the mechanism of a non-minimal curvature?matter coupling. An interesting feature of this gravitational theory is that the divergence of the energy-momentum tensor is nonzero. Firstly, they have reformulated the model in terms of an equivalent scalar-tensor theory, with two arbitrary potentials. By using the formalism of open thermodynamic systems, they have interpreted the energy balance equations in this gravitational theory from a thermodynamic point of view, as describing irreversible matter creation processes. The particle number creation rates, the creation pressure, and the entropy production rates have explicitly obtained as functions of the scalar field and its potentials, as well as of the matter Lagrangian. The temperature evolution laws of the newly created particles are also obtained. The cosmological implications of the model have briefly investigated, and it is shown that the late-time cosmic acceleration may be due to particle creation processes. Furthermore, it has also shown that due to the curvature-matter coupling, during the cosmological evolution a large amount of comoving entropy is also produced.

Mitra et al. [74] have studied thermodynamics laws by assuming flat FRW universe enveloped by by apparent and event horizon in the framework of RSII brane model and DGP brane scenario. Assuming extended Hawking temperature on the horizon, the unified first law is examined for perfect fluid (with constant equation of state) and Modified Chaplygin Gas model. As a result there is a modification of Bekenstein entropy on the horizons. Further the validity of the generalized second law of ther- modynamics and thermodynamical equilibrium are also investigated.

Pan et al. [75] investigated the expansion of the universe powered by the gravitationally induced adiabatic matter creation by developing general creation rate and their dynamical analysis. They also developed dynamical analysis in the the presence of a non-singular universe (without the big bang singularity) with two successive accelerated phases, one at the very early phase of the universe (i.e. inflation), and the other one describes the current accelerating universe, where this early, late accelerated phases are associated with an unstable fixed point (i.e. repeller) and a stable fixed point (attractor), respectively.

Sal and Haro [76] performed a qualitative and thermodynamic study of two models when one takes into account adiabatic particle production. In the first one, there is a constant particle production rate, which leads to solutions depicting the current cosmic acceleration but without inflation. The other one has solutions that unify the early and late time acceleration. These solutions converge asymptotically to the thermal equilibrium.

Recently, by assuming the gravitationally induced particle scenario with constant specific entropy and arbitrary particle creation rate $(\Gamma)$, thermodynamics on the apparent horizon for FRW universe has been discussed [49]. They have investigated the first law, GSLT and thermodynamical equilibrium by assuming the EoS for perfect fluid and put forward various constraints on $\Gamma$ for which thermodynamical laws hold. We have extended the work of [49] in the DGP brane-world scenario by assuming usual entropy as well as its entropy corrections (power law as well as logarithmic corrected) in flat FRW universe. We have extracted EoS parameter and obtained its various constraints with respect to quintessence, vacuum and phantom era of the universe. For variable as well as constant particle creation rate $(\Gamma)$, the first law of thermodynamics, GSLT and thermal equilibrium condition is satisfied in all cases of entropies forms.

Open Access This article is distributed under the terms of the Creative Commons Attribution 4.0 International License (http://creativecomm ons.org/licenses/by/4.0/), which permits unrestricted use, distribution, and reproduction in any medium, provided you give appropriate credit to the original author(s) and the source, provide a link to the Creative Commons license, and indicate if changes were made. Funded by SCOAP ${ }^{3}$.

\section{References}

1. A.G. Riess et al., Astron. J. 116, 1009 (1998)

2. S. Perlmutter et al., Astrophys. J. 517, 565 (1999)

3. P.J.E. Peebles, B. Ratra, Rev. Mod. Phys. 75, 559 (2003)

4. S.W. Hawking, Commun. Math. Phys. 43, 199 (1975)

5. T. Jacobson, Phys. Rev. Lett. 75, 1260 (1995) 
6. T. Padmanabhan, Class. Quantum Grav. 19, 5387 (2002)

7. J.D. Bekenstein, Phys. Rev. D 7, 2333 (1973)

8. R.G. Cai, S.P. Kim, JHEP 02, 050 (2005)

9. R. Banerjee, S.K. Modak, JHEP 073, 0911 (2009)

10. H. Wei, Commun. Theor. Phys. 52, 743 (2009)

11. S. Banerjee, R.K. Gupta, A. Sen, JHEP 147, 1103 (2011)

12. A. Sheykhi, M. Jamil, Gen. Relativ. Gravit. 43, 2661 (2011)

13. K. Karami, S. Ghaffari, M.M. Soltanzadeh, Class. Quantum Grav. 27, 205021 (2010)

14. M.R. Setare, JCAP 01, 023 (2007)

15. A. Sheykhi, Class. Quantum Grav. 27, 025007 (2010)

16. M. Mazumder, S. Chakraborty, Gen. Relativ. Gravit. 42, 813 (2010)

17. M. Jamil, E.N. Saridakis, M.R. Setare, Phys. Rev. D 81, 023007 (2010)

18. K. Karami et al., JHEP 150, 1108 (2011)

19. K. Karami et al., Eur. Phys. Lett. 93, 29002 (2011)

20. K. Bamba, C.Q. Geng, S. Tsujikawa, Int. J. Mod. Phys. D 20, 1363 (2011)

21. K. Bamba, C.Q. Geng, S. Tsujikawa, Phys. Lett. B 688, 101 (2010)

22. J.R. Mureika, J.W. Moffat, M. Faizal, Phys. Lett. B 757, 528 (2016)

23. M. Sharif, S. Rani, Eur. Phys. J. Plus 128, 96 (2013)

24. D. Momeni, P.H.R.S. Moraes, R. Myrzakulov, Astrophys. Space Sci. 361, 228 (2016)

25. S. Rani, T. Nawaz, A. Jawad, Astrophys. Space Sci. 361, 285 (2016)

26. E. Schrodinger, Phys. 6, 899 (1939)

27. L. Parker, Phys. Rev. Lett. 21, 562 (1968)

28. L. Parker, Phys. Rev. 183, 1057 (1969)

29. N.D. Birrell, P.C.W. Davies, Quantum fields in curved space (Cambridge University Press, Cambridge, 1982)

30. V. Mukhanov, S. Winitzki, Introduction to quantum effects in gravity (Cambridge University Press, Cambridge, 2007)

31. L. Parker, D.J. Toms, Quantum field theory in curved spacetime: quantized field and gravity (Cambridge University Press, Cambridge, 2009)

32. I. Prigogine et al., Gen. Relativ. Gravit. 21, 767 (1989)

33. M.O. Calvao, J.A.S. Lima, I. Waga, Phys. Lett. A 162, 223 (1992)

34. J.A.S. Lima, M.O. Calvao, I. Waga, arXiv:0708.3397 [astro-ph]

35. J.A.S. Lima, A.S.M. Germano, Phys. Lett. A 170, 373 (1992)

36. W. Zimdahl, D. Pavon, Gen. Relativ. Gravit. 26, 1259 (1994)

37. J. Gariel, G. le Denmat, Phys. Lett. A 200, 11 (1995)

38. L.R.W. Abramo, J.A.S. Lima, Class. Quantum Gravity 13, 2953 (1996)

39. J.A.S. Lima, A.S.M. Germano, L.R.W. Abramo, Phys. Rev. D 53, 4287 (1996)

40. J.A.S. Lima, J.S. Alcaniz, Astron. Astrophys. 348, 1 (1999)

41. J.S. Alcaniz, J.A.S. Lima, Astron. Astrophys. 349, 729 (1999)

42. J.A.S. Lima, F.E. Silva, R.C. Santos, Class. Quantum Grav. 25, 205006 (2008)
43. G. Steigman, R.C. Santos, J.A.S. Lima, JCAP 06, 033 (2009)

44. J.A.S. Lima, J.F. Jesus, F.A.O. liveira, JCAP 11, 027 (2010)

45. S. Basilakos, J.A.S. Lima, Phys. Rev. D 82, 023504 (2010)

46. T. Shiromizu, K.I. Maeda, M. Sasaki, Phys. Rev. D 62, 024012 (2000)

47. P. Binetruy, C. Deffayet, D. Langlois, Nucl. Phys. B 565, 269 (2000)

48. P. Binetruy, C. Deffayet, U. Ellwanger, D. Langlois, Phys. Lett. B 477, 285 (2000)

49. S. Subhajit, M. Anindita, Eur. Phys. J. C 77, 196 (2017)

50. G. Dvali, G. Gabadadze, M. Porrati, Phys. Lett. B 485, 208 (2000)

51. C. Deffayet, G. Dvali, G. Gabadadze, Phys. Rev. D 65, 044023 (2002)

52. G. Dvali, G. Gabadadze, Phys. Rev. D 63, 065007 (2001)

53. S.L. Adler, Phys. Rev. Lett. 44, 1567 (1980)

54. C. Deffayet, Phys. Lett. B 502, 199 (2001)

55. K. Koyama, Class. Quant. Grav. 24, 231 (2007)

56. L. Parker, D.J. Toms, Quantum field theory in curved spacetime: quantized field and gravity (Cambridge Univ. Press, Cambridge, 2009)

57. I. Prigogine, J. Geheniau, E. Gunzig, P. Nardone, Gen. Relativ. Gravit 21, 767 (1989)

58. J.A.S. Lima, M.O. Calvao, I. Waga, arXiv:0708.3397 [astro-ph]

59. R. Bousso, Phys. Rev. D 71, 064024 (2005)

60. W. Zimdahl, Phys. Rev. D 61, 083511 (2000)

61. K.A. Miessner, Class. Quantum Grav. 21, 5245 (2004)

62. A. Ghosh, P. Mitra, Phys. Rev. D 71, 027502 (2004)

63. A. Chatterjee, P. Majumder, Phys. Rev. Lett. 92, 141301 (2004)

64. R. Banerjee, S.K. Modak, JHEP 0905, 063 (2009)

65. S.K. Modak, Phys. Lett. B 671, 167 (2009)

66. M. Jamil, M.U. Farooq, JCAP 03, 001 (2010)

67. H.M. Sadjadi, M. Jamil, Europhys. Lett. 92, 69001 (2010)

68. N. Radicella, D. Pavon, Phys. Lett. B 691, 121 (2010)

69. A. Sheykhi, M. Jamil, Gen. Rel. Grav. 43, 2661 (2011)

70. M.U. Farooq, M. Jamil, J. Canadian, Phys. 89, 1251 (2011)

71. K. Karami, A. Sheykhi, M. Jamil, S. Ghaffari, A. Abdolmaleki, arXiv:1106.2406v1 [physics.gen-ph]

72. S. Das, S. Shankaranarayanan, S. Sur, Phys. Rev. D 77, 064013 (2008)

73. T. Harko, F.S.N. Lobo, J.P. Mimoso, D. Pavon, Eur. Phys. J. C 75, $386(2015)$

74. S. Mitra, S. Saha, S. Chakraborty, Adv. High Energ. Phys. 2015, $430764(2015)$

75. S. Pan, J.D. Haro, A. Paliathanasis, R.J. Slagter, MNRAS 460, 14451456 (2016)

76. L.A. Sal, D. Haro, Class. Quantum Grav. 34, 065001 (2017) 